\title{
Quantitative Radiologic analysis and Clinical Outcomes of Urgent EC-IC Bypass for Hemodynamic Compromised Patients with Acute Ischemic Stroke
}

\section{Hyunjun Jo}

Korea University Ansan Hospital, Korea University College of Medicine

\section{Dongwook Seo}

Seoul National University Bundang Hospital, Seoul National University College of Medicine

\section{Young-Deok Kim}

Seoul National University Bundang Hospital, Seoul National University College of Medicine

\section{Seung Pil Ban}

Seoul National University Bundang Hospital, Seoul National University College of Medicine Tackeun Kim

Seoul National University Bundang Hospital, Seoul National University College of Medicine Oki Kwon

Seoul National University Bundang Hospital, Seoul National University College of Medicine

\section{Chang Wan Oh}

Seoul National University Bundang Hospital, Seoul National University College of Medicine

\section{Leonard Sunwoo}

Seoul National University

\section{Beom Joon Kim}

Seoul National University

\section{Moon-Ku Han}

Seoul National University

Hee-Joon Bae

Seoul National University

Si Un Lee ( $\sim$ nsmidget@gmail.com )

Seoul National University Bundang Hospital, Seoul National University College of Medicine Jae Seung Bang

Seoul National University Bundang Hospital, Seoul National University College of Medicine

\section{Research Article}


Keywords: urgent EC-IC bypass, STA-MCA bypass, acute ischemic stroke with large vessel occlusion, perfusion $\mathrm{CT}$, quantitative analysis

Posted Date: September 2nd, 2021

DOI: https://doi.org/10.21203/rs.3.rs-841902/v1

License: (c) (1) This work is licensed under a Creative Commons Attribution 4.0 International License. Read Full License 


\section{Abstract \\ Objective}

The treatment of choice for acute ischemic stroke with large vessel occlusion is intra-arterial thrombectomy. The extracranial-to-intracranial (EC-IC) bypass was relatively devaluated; however, recent studies have shown that this surgery can be helpful when performed for a highly selective patient group.

\section{Methods}

We analyzed 41 patients who underwent urgent EC-IC bypass at the Seoul National University Bundang Hospital after being selected using strict operation criteria and retrospectively reviewed the effectiveness and safety of the surgery. In particular, quantitative data from perfusion computed tomography images were reconstructed to analyze the changes in pre- and postoperative perfusion status in terms of objective numerical values. We also clinically assessed the patients' neurological outcome and complication rates.

\section{Results}

Immediate postoperatively, the volume of time-to-max (Tmax) $>6$ second decreased significantly; it continued to improve significantly until 6 months postoperatively (preoperative, $89 \mathrm{ml}$; immediate postoperative, $21.5 \mathrm{ml}$; postoperative 6 months, $10.5 \mathrm{ml}$ ). Ischemic core-penumbra mismatch volumes were also significantly improved until 6 months postoperatively (preoperative, $77 \mathrm{ml}$; immediate postoperative, $21 \mathrm{ml}$; postoperative 6 months, $5.5 \mathrm{ml}$ ). Other perfusion parameters, such as Tmax $>10 \mathrm{~s}$, Tmax $>8 \mathrm{~s}$, and Tmax $>4 \mathrm{~s}$ also showed statistically significant improvement. In addition, the patient's neurological condition significantly improved. The surgical complication rate in this study was similar to those in previous studies. The predictors of good postoperative neurological condition were the patient's preoperative condition and perfusion status of the patient immediately postoperatively.

\section{Conclusion}

Emergent EC-IC bypass can be useful for patients with acute ischemic stroke with large vessel occlusion ineligible for IAT if surgery is performed well after applying the strict surgical indications.

\section{Introduction}

Treatment of cerebral infarction has been rapidly progressing in recent decades. Currently, intra-arterial thrombectomy (IAT) is the treatment of choice for patients with cerebral infarction with large vessel occlusion within 24 hours of symptom onset ${ }^{1,2}$. In contrast, extracranial-to-intracranial (EC-IC) bypass has been relatively unacknowledged as it showed no benefit over medical treatment in two randomized 
controlled trials, the EC-IC Bypass Trial (EIBT) and the Carotid Occlusion Surgery Study (COSS) 3,4,5,6. However, despite recent rapid technological and instrumental advances, IAT still has a relatively high probability of failure and contraindication of about $10-20 \%$ of all cases ${ }^{1,2}$. In such cases, according to evidence-based medicine at present, the only possible treatment is medical therapy including induced hypertension treatment and dual antiplatelet therapy.

However, EC-IC bypass is reportedly effective in a highly selective patient group ${ }^{7-11}$. Researchers mainly performed surgery on IAT-ineligible patients, asserting the patients' good postoperative condition and preventing recurrent stroke. However, because this type of surgery has not yet been widely recognized, these studies had limitations, such as a small sample size and a short follow-up period. In addition, it is not easy to objectively prove the patients' radiological or clinical improvement because it is difficult to set the control group, which is another limitation. Thus, we analyzed the follow-up observation results of patients who underwent surgery for more than 1 year, on average, and quantitatively analyzed whether surgical treatment helps in improving the perfusion status in patients with cerebral infarction.

\section{Materials And Methods Operation indication}

Our hospital's institutional review board approved this retrospective study (B-2102/666 - 105). The requirement to obtain informed consent from the patients was waived. We performed urgent superficial temporal artery to middle cerebral artery (STA-MCA) bypass for acute ischemic stroke with intracranial stenosis or occlusion for patients with the following indications: 1 ) hemodynamic cerebral infarction on computed tomography (CT) scan or magnetic resonance imaging (MRI) with perfusion delay on imaging; 2) diffusion-perfusion mismatch on examination;3) neurological deterioration despite receiving the best medical treatment for cerebral infarction including antiplatelet therapy and induced hypertension at the neurology department; and 4) ineligible for intra-arterial thrombolysis, including failed intervention and contraindicative to IAT. Patients were usually admitted to a neurology department first; when the patient's neurological symptoms worsened despite the best medical treatment, we thought that the patient was not just beyond the timeframe from the infarction onset but had an on-going infarction for which revascularization would be helpful. If other treatments, such as intra-arterial thrombolysis, were ineligible, the patients were transferred to the neurosurgery department to undergo bypass. When the patient was determined to fulfill the abovementioned criteria, surgery was performed as soon as possible; all patients underwent surgery within 2 weeks of the first symptom onset.

\section{Surgical procedure}

Under general anesthesia, the parietal or frontal branch of the STA was dissected and used as the donor artery. We performed craniotomy around the hypoperfused area so blood flow could be supplied to the location of perfusion delay. Nevertheless, the vessel supplying blood to the eloquent area was not bypassed considering the possibility of complications. As a result, most of the craniotomy was performed 
around the Chater's point, which is likely to have blood vessels such as angular, supramarginal, and posterior temporal arteries. After an approximately 5-cm-diameter craniotomy was performed, the STA and distal MCA (M4) branch were anastomized with 8-10 stitches using prolene $10-0$. Then, intraoperative STA flow was measured using an ultrasonic flow meter. As the surgery was performed on patients who could not undergo preoperative medical therapy, dual antiplatelet therapy was maintained intraoperatively, and aspirin was resumed from the day after surgery. Intensive care was administered intra- and postoperatively to avoid lowering blood pressure (>120 $\mathrm{mmHg})$. When the required blood flow was observed to be sufficiently large, a double-barrel bypass was performed.

\section{Radiologic analysis}

All patients who underwent operation were examined using perfusion imaging techniques, such as perfusion computed tomography (CTP), within 24 hours preoperatively. These were also performed within 48 hours and 6 months postoperatively. Some patients were unstable immediately postoperatively and were not examined immediately postoperatively for intensive care. In some cases, perfusion examination was not performed at 6 months due to follow-up loss. Such patients were excluded from the analysis.

We processed the CTP data of some patients using RAPID (iSchemaView, Menlo Park, California, the USA), an automated perfusion measurement tool, without relying on the interpretation of the imaging studies, which is unavoidably subjective, and quantitatively evaluated their perfusion status ${ }^{12}$. CTPs was performed using a 256-slice CT scanner (Brilliance, Philips Medical Systems, Best, The Netherlands), and changes in time-to-maximum (Tmax) > $10 \mathrm{~s},>8 \mathrm{~s},>6 \mathrm{~s}$, and $>4 \mathrm{~s}$ on time-to-peak (TTP) were analyzed.

Cerebral blood flow $(\mathrm{CBF})<30 \%$, representing the volume of the ischemic core ${ }^{13}$, was also analyzed. Tmax $>6$ s represents penumbra ${ }^{13}$; therefore, the difference between Tmax $>6 \mathrm{~s}$ and $\mathrm{CBF}<30 \%$ was defined as the diffusion-perfusion mismatch volume, and this volume was also analyzed.

One week after the operation, all patients underwent transfemoral cerebral angiography (TFCA) to confirm the efficacy of the bypass patency.

\section{Clinical outcomes}

To analyze the patient's clinical results, preoperative modified Rankin Scale (mRS) and the National Institutes of Health Stroke Scale (NIHSS) were used, and the results were compared with NIHSS at discharge and mRS at last follow-up. Postoperatively, mRS of $\leq 2$ was defined as good outcome and $\geq 3$ was defined as a bad outcome. We defined a new infarction showing no specific symptoms but accidently identified as a silent infarction. If a new infarction accompanied by symptoms was identified on the ipsilateral side of the surgery postoperatively during hospital stay, it was defined as infarction progression. Neurological symptoms being induced in the hemisphere on the side of the surgery was performed and confirmed radiologically > 6 months postoperatively was defined as stroke recurrence.

\section{Statistical analysis}

Statistical analysis was performed using IBM SPSS statistics 25 (SPSS, Chicago, Illinois). Continuous variables were presented as median (interquartile ranges). The Wilcoxon matched-pairs signed-rank test 
was used to compare perfusion data before and after bypass, and the Friedman test was used to compare the data at three time points, including perfusion data at 6 months postoperatively. Univariate analysis was performed to see if there are factors predicting good outcome. In addition, to confirm if there was a significant difference in the perfusion parameters of the good outcome and the bad outcome groups, we used Mann-Whitney U-test. Generally, a $P$ value of $<0.05$ was considered as significant in this study.

\section{Results}

\section{Baseline characteristics}

From January 2006 to December 2020, we performed urgent STA-MCA bypass on 79 hemispheres of 78 patients with acute ischemic stroke and intracranial arterial stenosis or occlusion with cerebral infarction. Among them, 42 hemispheres of 41 patients had the pre- and postoperative quantitative perfusion data; only these patients were analyzed in this paper (Fig. 1). The median age of the patients was 66.5 (61.75$72.25)$ years, and it took $3(0-5)$ days from first symptom onset to hospitalization. Neurologists classified the patients according to the Trial of Org 10172 in Acute Stroke Treatment (TOAST) classification; 35 (83.3\%) patients were classified as having large-artery atherosclerosis (LAA), 3 (7.1\%) as having cardioembolism (CE), and it was not clear whether they had LAA or CE in $4(9.5 \%)$ patients. All seven patients also had large vessel atherosclerosis. Preoperative perfusion examination of the patients showed Tmax $>10 \mathrm{~s}$ of $24 \mathrm{ml}$ (14-65), Tmax > $8 \mathrm{~s}$ of $89 \mathrm{ml}$ (24-101), Tmax $>6 \mathrm{~s}$ of $146 \mathrm{ml}(67-212)$, Tmax $>4 \mathrm{~s}$ of $299 \mathrm{ml}(164-344), \mathrm{CBF}<30 \%$ of $0 \mathrm{ml}(0-11)$, and mismatch volume of $123 \mathrm{ml}(63-212)$ along with hemodynamic compromise. Moreover, 10 (23.8\%) of the 42 hemispheres underwent IAT but failed, whereas the remaining $32(76.2 \%)$ were not indicated for IAT. Of these, 22 patients were beyond the timeframe, 9 did not undergo IAT due to the high risk considering their vascular status, and 1 was not indicated for IAT because the symptoms were not severe. It took 4 (3-11.25) days from the first symptom onset to surgery. The location of the lesion, the number of anastomosis performed, and the preoperative $\mathrm{mRS}$ and NIHSS distribution are presented in the Table 1. 
Age (median (interquartile))

Sex (male, number (\%) / female, number (\%))

Side (right, number (\%) / left, number (\%))

Medical history, number (\%)

Hypertension

Diabetes mellitus

Hyperlipidemia

Onset to hospitalization, days (median (interquartile))

Onset to operation, days (median (interquartile))

Follow-up period, months (median (interquartile))

TOAST classification, number (\%)

Large-artery atherosclerosis

Cardioembolism

Not clear whether LAA or CE

Location of lesion, number (\%)

Proximal ICA

Distal ICA

M1

M2

Lesion severity, number (\%)

Occlusion

Severe stenosis

Number of anastomosis, number (\%)

Double barrel

Single barrel

Pre-operative mRS, number (\%)

*TOAST = Trial of Org 10172 in Acute Stroke Treatment; LAA = large-artery atherosclerosis; $C E=$ cardioembolism; ICA = internal carotid artery; mRS = modified Rankin Scale; NIHSS = National Institute of Health Stroke Scale
$66.5(61.75-72.25)$

$29(69.0) / 13(31.0)$

17 (40.5) / 25 (59.5)

$27(64.3)$

$14(33.3)$

$16(38.1)$

$3(0-5)$

$4(3-11.25)$

11.7 (3.85-20.98)

35 (83.3)

$3(7.1)$

4 (9.5)

18 (42.9)

6 (14.3)

$15(35.7)$

$3(7.1)$

$37(88.1)$

5 (11.9)

7 (16.7)

35 (83.3) 


\begin{tabular}{|ll|}
\hline Age (median (interquartile)) & $66.5(61.75-72.25)$ \\
\hline 0 & $2(4.8)$ \\
\hline 1 & $2(4.8)$ \\
\hline 2 & $5(11.9)$ \\
\hline 3 & $9(21.4)$ \\
\hline 4 & $16(38.1)$ \\
\hline 5 & $8(19.0)$ \\
\hline Pre-operative NIHSS (median (interquartile)) & $9(5.75-12)$ \\
\hline $\begin{array}{l}* \text { TOAST = Trial of Org 10172 in Acute Stroke Treatment; LAA = large-artery atherosclerosis; CE = } \\
\text { cardioembolism; ICA = internal carotid artery; mRS = modified Rankin Scale; NIHSS = National Institute } \\
\text { of Health Stroke Scale }\end{array}$ \\
\hline
\end{tabular}

\section{Radiologic analysis}

Quantitative assessment of immediate postoperative perfusion status

We quantitatively analyzed 42 patients whose data of CTP could be reconstructed with RAPID. Preoperatively and immediately postoperatively, Tmax $>10 \mathrm{~s}$ was $13 \mathrm{ml}(0-36.25)$ and $0 \mathrm{ml}(0-3.5)$ respectively, showing a statistically significant difference $(p=.000)$. Tmax $>8 \mathrm{~s}$ also showed a significant difference of $34 \mathrm{ml}(13.5-90.25)$ and $0 \mathrm{ml}(0-7)$ preoperative and postoperatively. Both Tmax $>6 \mathrm{~s}(83 \mathrm{ml}$ [53.75-168.75] to $21 \mathrm{ml}[2.25-48.75], \mathrm{p}=.000)$ and Tmax > $4 \mathrm{~s}(216.5 \mathrm{ml}$ [155.25-296] to $140.5 \mathrm{ml}$ [64.75-226.75], $p=.000$ ) also improved significantly. The pre- and postoperative volume of CBF $<30 \%$ decreased from $0 \mathrm{ml}(0-8.75)$ to $0 \mathrm{ml}(0-0)$, which was statistically significant $(p=.014)$. The diffusionperfusion mismatch volume was calculated as the difference between Tmax $>6 \mathrm{~s}$ and $\mathrm{CBF}<30 \%$, and it decreased significantly from $74.5 \mathrm{ml}(40.5-155.5)$ to $20.5 \mathrm{ml}(0-48.75 ; \mathrm{p}=.000)$ (Table 2$)$. 
Table 2

Short term (pre- versus immediate post-operative) and long term (pre-, immediate post-operative and postoperative 6 months) comparison of CTP quantitative data (median (interquartile))

\begin{tabular}{|c|c|c|c|c|}
\hline CTP parameter & \multicolumn{2}{|l|}{ preOp } & postOp 0 & p-value \\
\hline $\operatorname{Tmax}>10 \mathrm{~s}(\mathrm{ml})$ & \multicolumn{2}{|c|}{$13(0-36.25)$} & $0(0-3.5)$ & .000 \\
\hline $\operatorname{Tmax}>8 \mathrm{~s}(\mathrm{ml})$ & \multicolumn{2}{|c|}{$34(13.5-90.25)$} & $0(0-7)$ & .000 \\
\hline $\operatorname{Tmax}>6 s(\mathrm{ml})$ & \multicolumn{2}{|c|}{$83(53.75-168.75)$} & $21(2.25-48.75)$ & .000 \\
\hline $\operatorname{Tmax}>4 s(\mathrm{ml})$ & \multicolumn{2}{|c|}{$216.5(155.25-296)$} & $140.5(64.75-226.75)$ & .000 \\
\hline $\mathrm{CBF}<30 \%(\mathrm{ml})$ & \multicolumn{2}{|c|}{$0(0-8.75)$} & $0(0-0)$ & .014 \\
\hline Mismatch volume (ml) & \multicolumn{2}{|c|}{$74.5(40.5-155.5)$} & $20.5(0-48.75)$ & .000 \\
\hline \multicolumn{5}{|c|}{ Long term comparison of CTP quantitative data $(n=31)$} \\
\hline CTP parameter & preOp & postOp 0 & postOp 6 & $\begin{array}{l}\mathrm{p}- \\
\text { value }\end{array}$ \\
\hline $\operatorname{Tmax}>10 \mathrm{~s}(\mathrm{ml})$ & $12(0-34)$ & $0(0-6)$ & $0(0-0)$ & .000 \\
\hline $\operatorname{Tmax}>8 \mathrm{~s}(\mathrm{ml})$ & $27(15-89)$ & $0(0-9)$ & $0(0-8)$ & .000 \\
\hline $\operatorname{Tmax}>6 \mathrm{~s}(\mathrm{ml})$ & $78(61-159)$ & $23(0-65)$ & $7(0-22)$ & .000 \\
\hline $\operatorname{Tmax}>4 s(\mathrm{ml})$ & $\begin{array}{l}219(164- \\
281)\end{array}$ & $150(79-236)$ & $118(43-223)$ & .000 \\
\hline $\mathrm{CBF}<30 \%(\mathrm{ml})$ & $0(0-12)$ & $0(0-0)$ & $0(0-5)$ & .023 \\
\hline $\begin{array}{l}\text { Mismatch volume } \\
(\mathrm{ml})\end{array}$ & $72(47-148)$ & $23(0-57)$ & $5(0-17)$ & .000 \\
\hline
\end{tabular}

${ }^{*} \mathrm{CTP}=$ perfusion computed tomography; preOp = pre-operative; postOp 0 = immediate post-operative; Tmax = time-to-maximum; $\mathrm{CBF}=$ cerebral blood flow

Quantitative assessment of long-term postoperative perfusion status

We performed a follow-up perfusion examination for 31 of our patients at 6 months postoperatively and analyzed the results. The value of $\operatorname{Tmax}>10 \mathrm{~s}$ showed a continuous decreasing trend as preoperative, 12 $\mathrm{ml}(0-34)$; immediate postoperative, $0 \mathrm{ml}(0-6)$; postoperative 6 months, $0 \mathrm{ml}(0-0)$, which was statistically significant $(p=.000)$. Tmax $>8 \mathrm{~s}$ also continued to decrease as preoperative, $27 \mathrm{ml}(15-89)$; immediate postoperative, $0 \mathrm{ml}(0-9)$; postoperative 6 months, $0 \mathrm{ml}(0-8)(p=.000)$. Tmax $>6 \mathrm{~s}$ (preoperative, $78 \mathrm{ml}$ [61-159]; immediate postoperative, $23 \mathrm{ml}$ [0-65]; postoperative 6 months, $7 \mathrm{ml}$ [022]; $p=.000$ ) and Tmax > $4 \mathrm{~s}$ (preoperative, $219 \mathrm{ml}$ [164-281; immediate postoperative, $150 \mathrm{ml}$ [79-236]; postoperative 6 months, $118 \mathrm{ml}$ [43-223], $\mathrm{p}=.000$ ) also showed statistically significant decreases. The $\mathrm{CBF}<30 \%$ value showed a trend of preoperative, $0 \mathrm{ml}(0-12)$; immediate postoperative, $0 \mathrm{ml}(0-0)$; 
postoperative 6 months, $0 \mathrm{ml}(0-5)$, which was statistically significant $(p=.023)$. The mismatch volume calculated by the above method was preoperative, $72 \mathrm{ml}(47-148)$; immediate postoperative, $23 \mathrm{ml}$ (057); postoperative 6 months, $5 \mathrm{ml}(0-17)$, showing a statistically significant decrease $(p=.000)$ (Table 2$)$. Figure 2 shows these figures as a graph.

Transfemoral cerebral angiography

All patients showed good bypass patency, and it was confirmed that the flow spread well to intracranial vessels through the STA. No occlusion or stenosis site was recanalized.

\section{Clinical outcomes}

We followed up patients for 11.7 months (3.85-20.98). Figure 3 show the changes in mRS before and at the last follow-up. There were 27 patients $(64.3 \%)$ with mRS of 2 or less, which can be called a good outcome. This is an increase compared to 9 patients $(21.4 \%)$ preoperatively. Comparing the preoperative NIHSS and the NIHSS at discharge, it was found that there was a significant improvement from 9 (5.7512) to $4(1-6.25)(p=.000)$.

\section{Outcome analysis}

In univariate analysis, preoperative mRS and preoperative NIHSS were found as preoperative factors that influence the good postoperative outcome. CTP parameter was not a factor influencing good outcomes (Table 3). Among all preoperative factors, there were only a few definite factors affecting the postoperative outcome of patients, so we performed Mann-Whitney U-test by dividing the patients into those with good and bad outcomes to find out if there were any significantly different CTP parameters between the two groups. As a result, preoperative mismatch volume $(p=.044)$, immediately postoperative Tmax $>6 s(p=.027)$, Tmax $>4 s(p=.039)$ and mismatch volume $(p=.020)$ were found to be significantly different in the good and bad outcome groups. Using T-test for these parameters, we found significant differences in the two groups in all three parameters as shown in Table 4. We identified the area under the curve (AUC) by drawing the receiver operating characteristic (ROC) curve for the three parameters (Fig. 4). The AUC was 0.712, 0.741, 0.723, and 0.760 , and the cut-off values were $58.5 \mathrm{ml}, 22.5 \mathrm{ml}, 165 \mathrm{ml}$, and $22.5 \mathrm{ml}$, respectively. 
Table 3

Univariate analysis of factors affecting good outcome

\begin{tabular}{|lc|}
\hline Factor & Univariate analysis p-value \\
\hline Age & .107 \\
\hline Onset to operation & .577 \\
\hline Location of lesion & .684 \\
\hline Lesion severity & .999 \\
\hline Number of anastomosis & .221 \\
\hline preOp mRS & .010 \\
\hline preOp NIHSS & .001 \\
\hline preOp Tmax $>10 s$ & .070 \\
\hline preOp Tmax $>8 s$ & .070 \\
\hline preOp Tmax $>6 s$ & .150 \\
\hline preOp Tmax $>4 s$ & .297 \\
\hline preOp CBF $<30 \%$ & .123 \\
\hline preOp mismatch volume & .289 \\
\hline *preOp = pre-operative; mRS = modified Rankin Scale; NIHSS = National Institutes of Health Stroke \\
\hline Scale; Tmax = time-to-maximum; CBF = cerebral blood flow
\end{tabular}


Table 4

The comparison of pre- and immediate post-operative CTP parameters of good outcome group and bad outcome group (median (interquartile))

\begin{tabular}{|c|c|c|c|}
\hline & Good outcome & Bad outcome & p-value \\
\hline preOp Tmax > 10s (ml) & $8(0-34)$ & $23(4-60)$ & .387 \\
\hline preOp Tmax > 8s (ml) & $27(6-56)$ & $50(19-119)$ & .123 \\
\hline preOp Tmax > 6s (ml) & $75(30-120)$ & $150(63-209)$ & .054 \\
\hline preOp Tmax > 4s (ml) & $192(132-268)$ & $281(179-344)$ & .155 \\
\hline preOp CBF $<30 \%(\mathrm{ml})$ & $0(0-12)$ & $0(0-8)$ & .611 \\
\hline preOp mismatch volume (ml) & $56(13-120)$ & $125(63-209)$ & .044 \\
\hline post0p 0 Tmax > 10s (ml) & $0(0-0)$ & $0(0-10)$ & .183 \\
\hline postOp 0 Tmax $>8 s(\mathrm{ml})$ & $0(0-5)$ & $3(0-36)$ & .178 \\
\hline postOp 0 Tmax $>6 s(\mathrm{ml})$ & $11(0-35)$ & $48(23-100)$ & .027 \\
\hline postOp 0 Tmax $>4 s(\mathrm{ml})$ & $124(57-157)$ & $224(173-259)$ & .039 \\
\hline postOp 0 CBF $<30 \%(\mathrm{ml})$ & $0(0-0)$ & $0(0-0)$ & .088 \\
\hline postOp 0 mismatch volume (ml) & $11(0-35)$ & $48(23-100)$ & .020 \\
\hline
\end{tabular}

\section{Complications}

We analyzed all postoperative complications. Three $(7.1 \%)$ were cases of silent infarction on diffusion MR performed for routine follow-up without worsening of the symptoms. These 3 patients showed similar clinical course as those without silent infarction. There was one (2.4\%) case of infarction progression, even though the patient underwent bypass. On the second postoperative day, the patient complained of worsening weakness on the left side. Multiple new infarctions in the right parietal and temporal cortex were identified on the diffusion MRI. The patient finally recovered just as well as other patients. No patients showed stroke recurrence.

There was one case of postoperative epidural hematoma after double-barrel bypass. After emergent hematoma removal surgery, the patient recovered well. In addition, there was one patient who underwent reoperation due to an infection at the surgical site. No patient died from cerebral complications.

\section{Discussion}

We demonstrated in our previous study that advancing imaging techniques have made it possible to select a target treatment for patients with acute ischemic stroke ${ }^{11}$. Since our previous study, a series of 
studies have been published to discuss the effect of EC-IC bypass. Horiuchi et al. suggested that EC-IC bypass improves neurological function in approximately $70 \%$ of patients ${ }^{10}$. Gunawardena et al. argued that hemodynamic insufficiency could be a rationale for EC-IC bypass ${ }^{8}$. Very recently, Kim et al. integrated the results of recent EC-IC bypass papers through pooled analysis and demonstrated that EC-IC bypass may be helpful in IAT-ineligible patients $7,11,14-21$. Unlike earlier studies, recent papers that performed surgical reperfusion in patients with acute ischemic stroke present promising results.

In our opinion, the difference between previous and recent studies lies in patient selection and operation criteria; there have been studies criticizing this point of the EIBT and COSS studies ${ }^{22-26}$. Moreover, surgical tools have evolved and surgical skills have advanced since these two studies. Therefore, it is reasonable to re-evaluate the role of EC-IC bypass in the treatment of acute cerebral infarction with large vessel occlusion.

In particular, in recent years, several studies have been conducted on perfusion examination using CT and MRI as well as the importance of each parameter. American Heart Association (AHA) analyzed existing studies to identify how to comprehend perfusion studies in clinical practice in $2020^{13}$. They reviewed the literature on how it is reasonable to judge salvageable tissue or penumbra. They also argued that as perfusion imaging techniques develop, it is possible to identify patients who will benefit from reperfusion from among patients who are beyond the conventional timeframe. Furthermore, as the collateral channel can be different for each patient, they also insisted that individualized treatment is possible when using a perfusion examination rather than simply taking the time from symptom onset.

In our study, some areas of hypoperfusion due to large vessel occlusion had already become ischemic cores, but there are cases where a significant area still had salvageable tissue, even after 24 hours, which is the IAT timeframe. The reason for symptom fluctuation despite the treatment for induced hypertension when IAT fails is probably because the perfusion state in the penumbra region repeats wax and wane. There have been studies showing that the patients' neurologic status is improved after EC-IC bypass, but this is the first study to quantitatively prove that the perfusion state improves when EC-IC bypass is performed in these cases.

In our study, immediately after EC-IC bypass, the Tmax was significantly improved in all sections. Assuming that the part of Tmax $>6 \mathrm{~s}$ minus $\mathrm{CBF}<30 \%$ is the penumbra, the size of the penumbra also significantly decreased pre- and postoperatively. These changes occurred between an examination performed at 24 hours preoperatively and 48 hours postoperatively; therefore, it is reasonable to assume that these changes were due to surgery rather than natural course of collateral vessel formation after cerebral infarction. Considering that there was no case of spontaneous reopening of occlusion or stenosis in TFCA performed 1 week postoperatively, it is more reasonable to argue that this change is due to surgery. Notably, CBF $<30 \%$, known to represent an ischemic core in several studies, have been shown to decrease slightly immediately postoperatively in our study. This is considered to be because in the acute ischemic state, cerebral edema occurs around the ischemic core; due to this, $\mathrm{CBF}<30 \%$ appeared in a wider range than the actual ischemic core. 
Only the preoperative neurological condition of the patient was found as the preoperative factor predicting good outcome. Among the CTP parameters, there was no factor that could predict good postoperative outcomes. This could be due to selection bias as this study includes patients who have been selected using strict operation criteria. Instead, in the postoperative comparison between the good and bad outcome groups, preoperative mismatch volume, immediate postoperative Tmax $>6 \mathrm{~s}$, Tmax $>4$ $\mathrm{s}$, and mismatch volume were significantly different. This means that the patient with a preoperative mismatch volume of $<58.5 \mathrm{ml}$ should undergo surgery, and the patient's mismatch volume must be clearly improved to $<22.5 \mathrm{ml}$ immediately postoperatively to achieve a good outcome. In other words, it is important that an experienced surgeon performs the surgery skillfully to obtain good prognosis. In all patients in our study, STA patency was well maintained in TFCA at postoperative 1 week. The high patency may be attributed to the difference in characteristics of the disease itself partly in light of the many experiences of moyamoya disease treatment at our hospital, but basically, the patient's perfusion status improves a lot when the bypass is well done.

Among the patients in this study, despite three silent infarctions, one infarction progression, and one postoperative hematoma, none of the patients showed postoperative deterioration. The infarction progression rate was $2.56 \%$, which is similar to recent studies 7,1016 . The proportion of patients with good postoperative outcomes was $61.5 \%$, which was also similar to other studies 7,158 . Notably, there was no patient with hyperperfusional hemorrhage in this study. Compared to the previous studies where the incidence of intracerebral hemorrhage (ICH) after IAT was approximately $5 \%$, it is estimated that the incidence of hyperperfusional hemorrhage is not high even though the operation was performed beyond the timeframe from the symptom onset. However, as the target groups are completely different, it is not reasonable to make a simple comparison. However, compared to antegrade recanalization, which enables a high flow to the area where the ischemic core has already been formed, the occurrence of hyperperfusional ICH may be relatively low if retrograde recanalization with lower flow by STA-MCA bypass is performed in blood vessels avoiding the ischemic core.

This study was conducted with larger number of patients than previous studies; however, there are still a few limitations. The number of patients is not absolutely large. In addition, it has a disadvantage of being a retrospective study without a control group. Furthermore, we failed to predict what factors should be preoperatively considered to make prognosis good. Further randomized controlled studies with a larger number of patients are warranted to judge whether urgent EC-IC bypass is actually effective in IATineligible acute ischemic stroke with large vessel occlusion patients.

\section{Conclusion}

For carefully selected IAT-ineligible hemodynamic compromised acute ischemic stroke with large vessel occlusion patients, emergent EC-IC bypass can be an alternative treatment that can improve not only the patient's perfusion status quantitatively radiologically but also the patient's neurological status clinically. The immediate postoperative perfusion status is correlated to the good outcome. 


\section{Declarations}

\section{Acknowledgement}

This work was supported by the 2020 Seoul National University Bundang Hospital research grant.

\section{Statement of Ethics}

The current study was approved by the institutional review board of the institution (B-2102/666-105). The Seoul National University Bundang Hospital Ethical Committee approved the study protocol and waived the requirement for individual patient consent to be provided for the study. This retrospective observational study involving human participants was in accordance with the ethical standards of the local ethics committee and with the 1964 Helsinki Declaration and its later amendments or comparable ethical standards.

\section{Conflict of Interest}

The authors have no personal financial or institutional interest in any of the drugs, materials, or devices described in this article

\section{Funding Source}

The authors did not receive any funding.

\section{Author Contributions}

All authors contributed to the study conception and design. Material preparation, data collection and analysis were performed by Hyunjun Jo and Dongwook Seo. The first draft of the manuscript was written by Hyunjun Jo and all authors commented on previous versions of the manuscript. All authors read and approved the final manuscript.

\section{References}

1. Albers, G. W. et al. Thrombectomy for Stroke at 6 to 16 Hours with Selection by Perfusion Imaging. $N$. Engl. J. Med, 378, 708-718 (2018).

2. Nogueira, R. G. et al. Thrombectomy 6 to 24 Hours after Stroke with a Mismatch between Deficit and Infarct. N. Engl. J. Med, 378, 11-21 (2018).

3. Farber, S., Diamond, L. K., Mercer, R. D., Sylvester, R. F. \& Wolff, J. A. Failure of extracranial-intracranial arterial bypass to reduce the risk of ischemic stroke. Results of an international randomized trial. Nejm, 313, 1191-1200 (1985).

4. Barnett, H. J. M. et al. Further conclusions from the extracranial-intracranial bypass trial. Surg. Neurol, 26, 227-235 (1986). 
5. Grubb, R. L. et al. Surgical results of the carotid occlusion surgery study. J. Neurosurg, 118, 25-33 (2013).

6. Powers, W. J. et al. Extracranial-intracranial bypass surgery for stroke prevention in hemodynamic cerebral ischemia: The carotid occlusion surgery study randomized trial. JAMA - J. Am. Med. Assoc, 306, 1983-1992 (2011).

7. Hun, J., Yoon, W., Kim, K. \& Jin, H. Efficacy and Safety of Timely Urgent Superficial Temporal Arteryto-Middle Cerebral Artery Bypass Surgery in Patients with Acute Ischemic Stroke: A SingleInstitutional Prospective Study and aPooled Analysis.08308, (2021).

8. Gunawardena, M., Rogers, J. M., Stoodley, M. A. \& Morgan, M. K. Revascularization surgery for symptomatic non-moyamoya intracranial arterial stenosis or occlusion. J. Neurosurg, 132, 415-420 (2020).

9. Komatani, H., Okamoto, Y., Aoki, T., Noguchi, K. \& Morioka, M. Long-term prognosis after extracranialintracranial bypass surgery for symptomatic cerebrovascular occlusive disease. Kurume Med. J, 64, 1-4 (2017).

10. Horiuchi, T. et al. Emergency EC-IC bypass for symptomatic atherosclerotic ischemic stroke. Neurosurg. Rev, 36, 559-565 (2013).

11. Hwang, G. et al. Superficial temporal artery to middle cerebral artery bypass in acute ischemic stroke and stroke in progress. Neurosurgery, 68, 723-729 (2011).

12. Laughlin, B. B., Chan, A., Tai, W. A. \& Moftakhar, P. RAPID Automated CT Perfusion in Clinical Practice. Neuroimaging38-55(2019).

13. Demeestere, J., Wouters, A., Christensen, S., Lemmens, R. \& Lansberg, M. G. Review of perfusion imaging in acute ischemic stroke: From time to tissue., 1017-1024 https://doi.org/10.1161/STROKEAHA.119.028337 (2020).

14. Nussbaum, E. S., Janjua, T. M., Defillo, A., Lowary, J. L. \& Nussbaum, L. A. Emergency extracranialintracranial bypass surgery for acute ischemic stroke. J. Neurosurg, 112, 666-673 (2010).

15. Lee, S. B. et al. Early superficial temporal artery to middle cerebral artery bypass in acute ischemic stroke. Clin. Neurol. Neurosurg, 115, 1238-1244 (2013).

16. Sugiyama, T. et al. Reappraisal of microsurgical revascularization for anterior circulation ischemia in patients with progressive stroke. World Neurosurg, 84, 1579-1588 (2015).

17. Burkhardt, J. K. et al. Emergency Extracranial-Intracranial Bypass to Revascularize Salvageable Brain Tissue in Acute Ischemic Stroke Patients. World Neurosurg, 109, e476-e485 (2018).

18. Kanematsu, R., Kimura, T., Ichikawa, Y. \& Inoue, T. Safety of urgent STA-MCA anastomosis after intravenous rt-PA treatment: a report of five cases and literature review. Acta Neurochir. (Wien), 160, 1721-1727 (2018).

19. Park, H. S. et al. Patient selection and clinical efficacy of urgent superficial temporal artery-middle cerebral artery bypass in acute ischemic stroke using advanced magnetic resonance imaging techniques. Oper. Neurosurg, 13, 552-559 (2017). 
20. Takeuchi, S. et al. Emergency superficial temporal artery to middle cerebral artery bypass after intravenous administration of tissue plasminogen activator for stroke. Turk. Neurosurg, 25, 633-637 (2015).

21. Inoue, A. et al. Efficacy of Early Superficial Temporal Artery-Middle Cerebral Artery Double Anastomoses for Atherosclerotic Occlusion in Patients with Progressing Stroke. J. Stroke Cerebrovasc. Dis, 26, 741-748 (2017).

22. Amin-Hanjani, S. et al. Extracranial-intracranial bypass for stroke - Is this the end of the line or a bump in the road? Neurosurgery, 71, 557-561 (2012).

23. Ausman, J. I. \& Diaz, F. G. Critique of the extracranial-intracranial bypass study. Surg. Neurol, 26, 218-221 (1986).

24. Carlson, A. P., Yonas, H., Chang, Y. F. \& Nemoto, E. M. Failure of cerebral hemodynamic selection in general or of specific positron emission tomography methodology? Carotid Occlusion Surgery Study (COSS)., 42, 3637-3639 (2011).

25. Goldring, S. \& Zervas, N. L. T. The extracranial-intracranial bypass study. A report of the committee appointed by the American Association of Neurological Surgeons to examine the study. Nejm, 316, 817-820 (1987).

26. Jr, S. T. Was the international randomized trial of extracranial-intracranial arterial bypass representative of the population at risk? Nejm, 316, 814-816 (1987).

\section{Figures}


Figure 1 Flow diagram of the enrolled patients

Total 1,091 patients underwent EC-IC bypass surgery

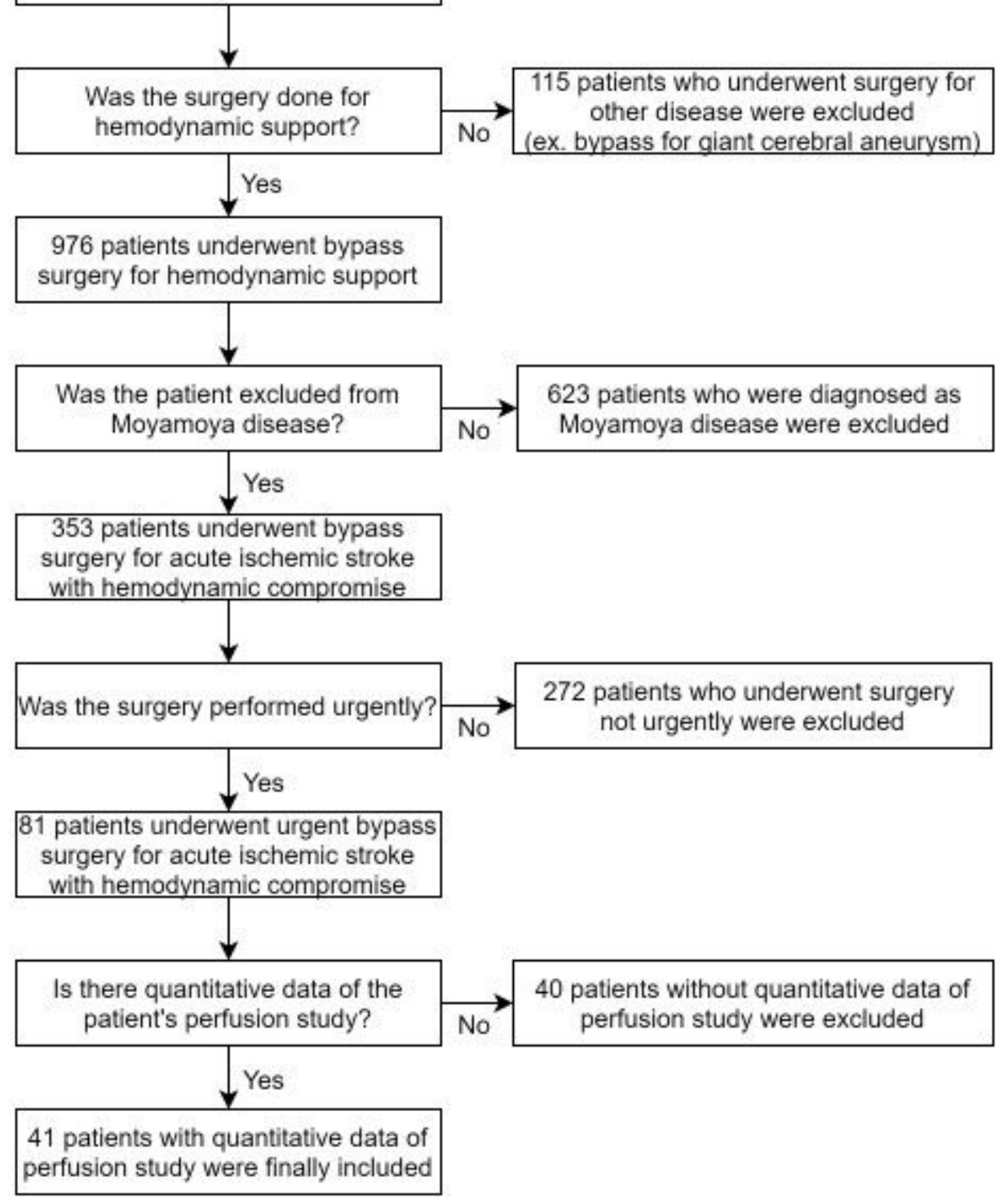

\section{Figure 1}

See image above for figure legend. 
Figure 2. Comparison of pre- and post-operative clinical outcomes

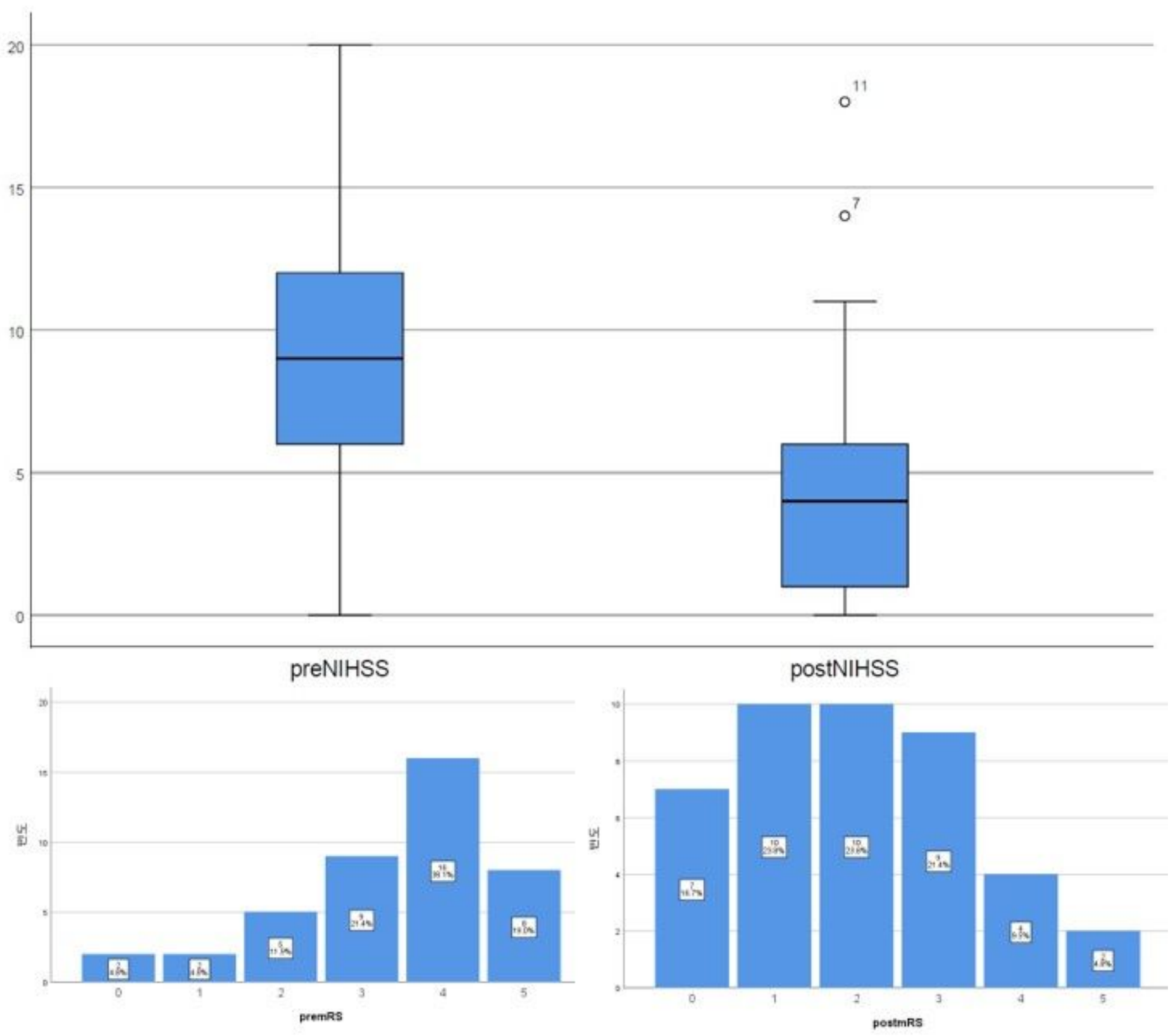

*preNIHSS $=$ pre-operative National Institute of Health Stroke Scale; postNIHSS $=$ National Institute of Health Stroke scale at discharge; premRS = pre-operative modified Rankin Scale; postmRS $=$ modified Rankin Scale at last follow-up

\section{Figure 2}

See image above for figure legend. 
Figure 3. Serial graph of pre-, immediate post-operative, and post-operative 6 month CTP quantitative data

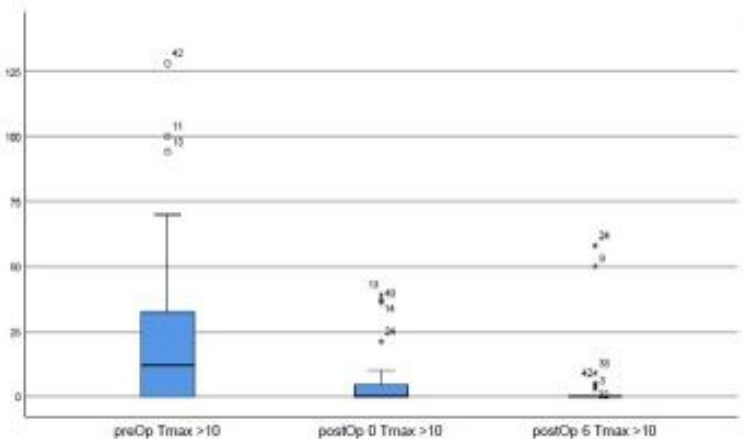

(a)

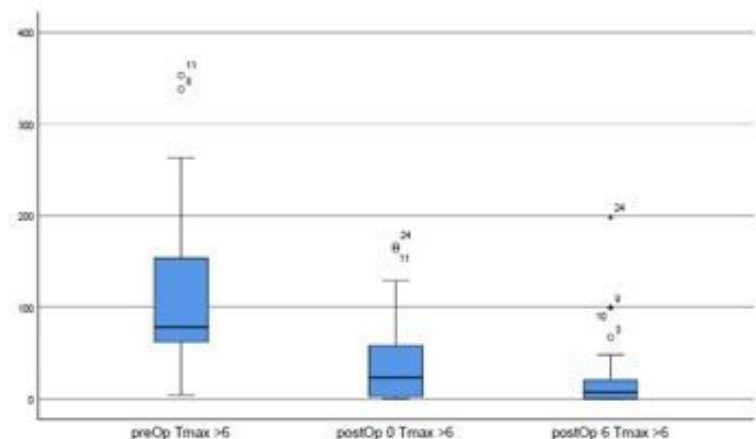

(c)

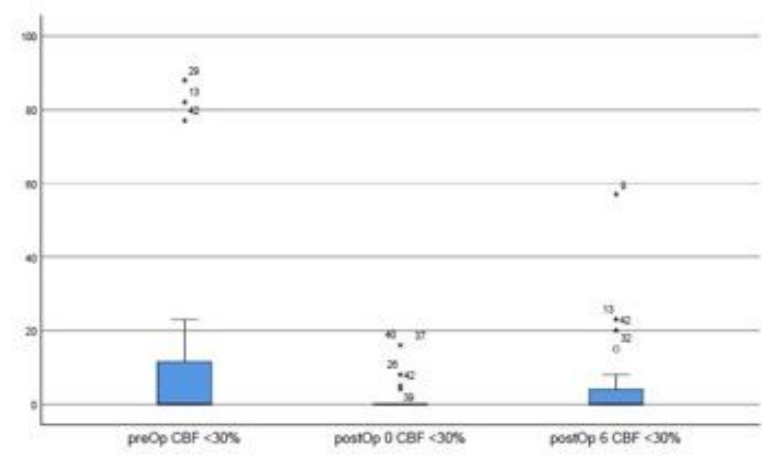

(e)

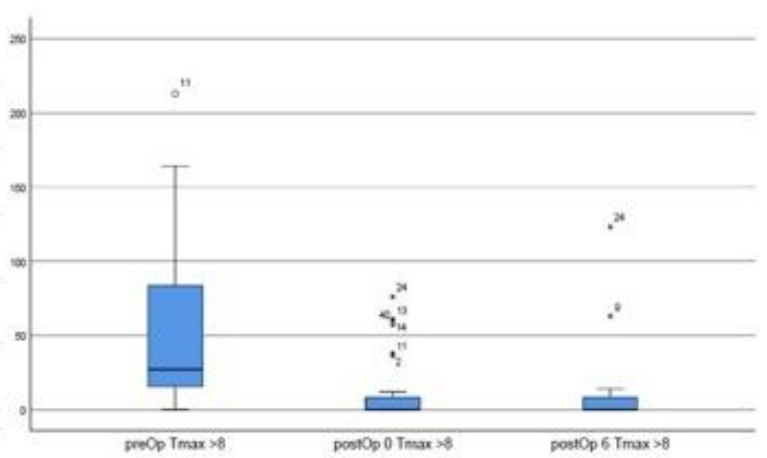

(b)

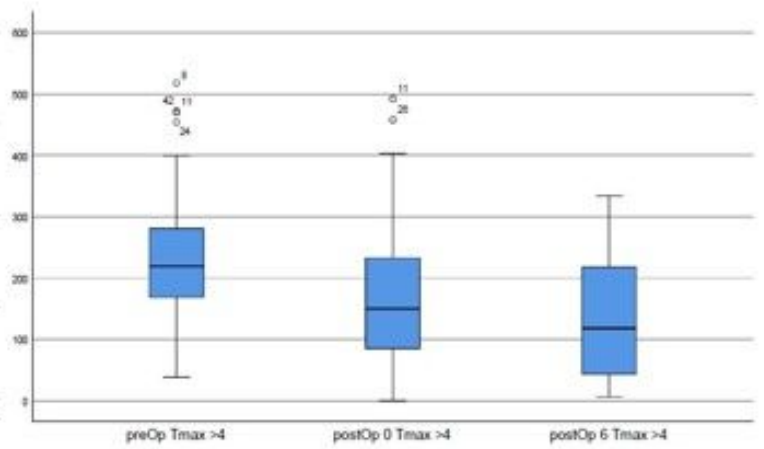

(d)

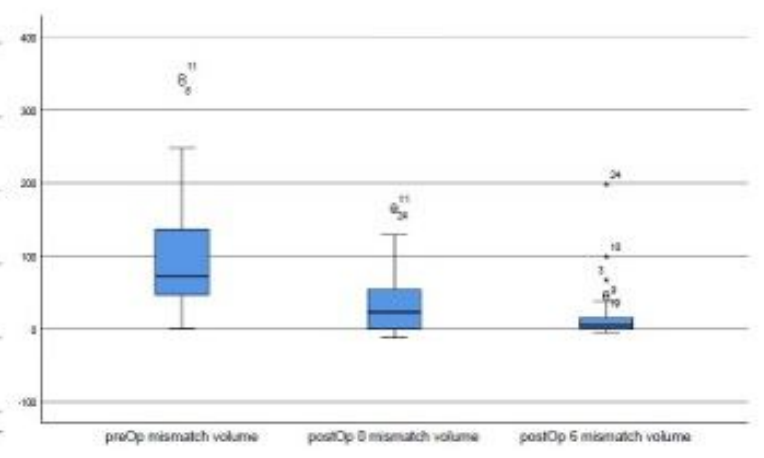

(f)

${ }^{*} \mathrm{CTP}=$ perfusion computed tomography; preOp $=$ pre-operative; postOp $0=$ immediate postoperative; postOp $6=$ post-operative 6 month; $\mathrm{Tmax}=$ time-to-maximum; $\mathrm{CBF}=$ cerebral blood flow

\section{Figure 3}

See image above for figure legend. 
Figure 4. ROC curve of (A) pre-operative mismatch volume, (B) immediate post-operative $T \max >6 s$, (C) immediate post-operative $T \max >4 s$ and (D) immediate post-operative mismatch volume

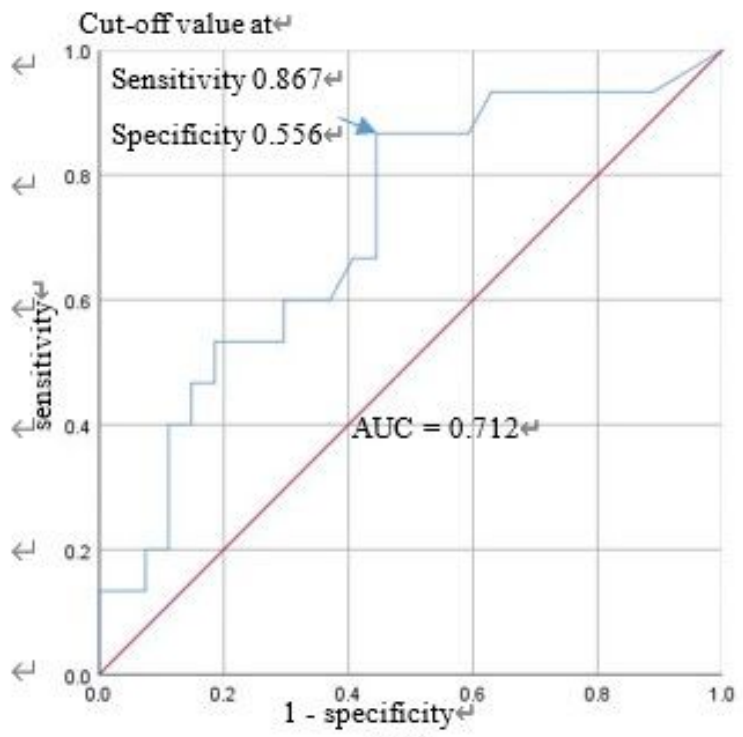

(A)

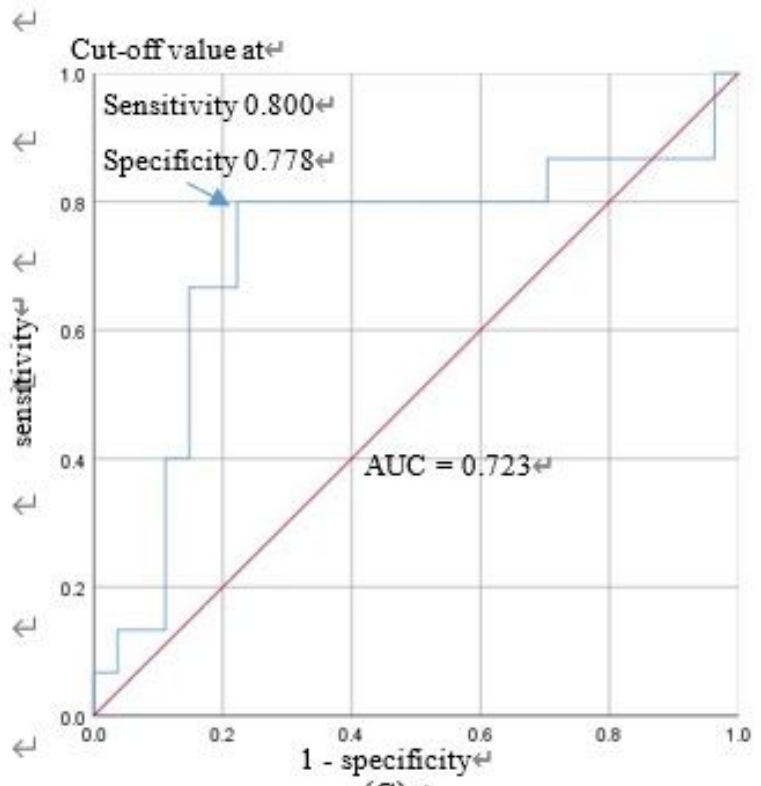

(C)

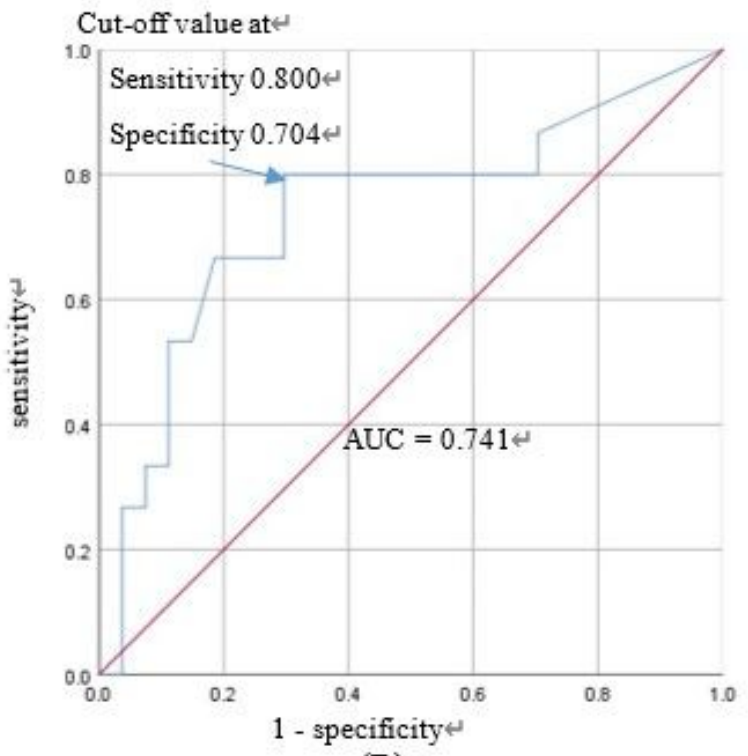

(B)

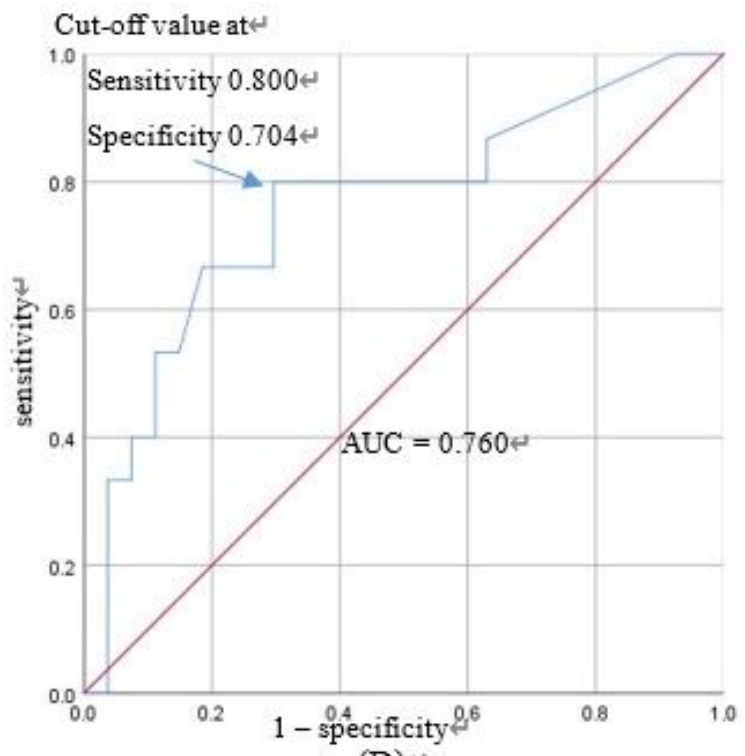

(D)

${ }^{*} \mathrm{ROC}=$ receiver operating characteristic; $\mathrm{AUC}=$ area under the curve; Tmax $=$ time-tomaximum

\section{Figure 4}

See image above for figure legend. 